Peer-Reviewed Article

ISSN: 2162-3104 Print/ ISSN: 2166-3750 Online

Volume 7, Issue 3 (2017), pp. 601-620

(C) Journal of International Students

http://jistudents.org/

doi: 10.5281/zenodo. 570021

\title{
The Relationship Between Second Language Anxiety and International Nursing Students Stress
}

\author{
Nigar G Khawaja \\ Sabrina Chan \\ Georgia Stein \\ Queensland University of Technology, Australia
}

\begin{abstract}
We examined the relationship between second language anxiety and international nursing student stress after taking into account the demographic, cognitive, and acculturative factors. International nursing students $(N=152)$ completed an online questionnaire battery. Hierarchical regression analysis revealed that spoken second language anxiety and the acculturative factors of marginalization and separation were significantly related to academic-related and placement-related stress in international nursing students. The results suggest that international nursing students, along with the pressures of their course, encounter second language anxiety particularly with reference to its spoken aspect. Subsequently, interventions aimed at building resilience in international nursing students should include coping and management of second language issues.
\end{abstract}

Keywords: acculturation, fear of negative evaluation, dysfunctional perfectionism, international nursing students stress, language proficiency, second language anxiety.

Due to the growing number of students who are choosing to study internationally, there has been an increased amount of research focusing on the wellbeing and stressors of the international student population. Research has found that there are many factors and variables that can influence the university experience of students and impact their levels of stress (Phakiti et al., 2013; Seyedfatemi et al., 2007). For example, all university students, including international students, can be influenced by various demographic factors. Cognitive factors, such as the fear of being negatively evaluated and 
displaying traits of perfectionism, are also factors that could influence the levels of stress experienced by university students. However, compared to domestic students, international students also have other factors that can contribute to the stress experienced at university. International students are affected by acculturative factors, such as marginalisation and separation, which have been found to be associated with higher levels of stress. Studies in this area have also indicated that language issues are a major source of concern for many international students, particularly speaking and writing in the host country's language, and these difficulties can often lead to students experiencing second language anxiety (SLA) (Brown, 2008; Fritz et al., 2008; Yu \& Shen, 2010).

SLA is a distinct type of anxiety experienced by individuals, who are using a second language. This second language is a new language acquired over and above the native language to function in a social, occupational or a professional setting (Brown, 2008). The proficiency of this second language may vary. SLA has primarily been conceptualized as consisting of spoken and written anxiety. The second language can be used to communicate with others. It can also be used to comprehend subject matter and to compose text. Anxiety can emerge in the form of emotional, social, physiological or cognitive symptoms. As a part of communicating in the second language, a person may experience fear, physiological arousal, and thoughts of being judged and evaluated by others. Writing in the second language can be a frustrating experience and can lead to fear of making mistakes, and evaluated by others as not good enough ( $\mathrm{Yu} \&$ Shen, 2010; Zheng, 2008).

While it is important to explore factors that contribute to international students' stress in general, international students studying nursing have the added stress of clinical placements, in addition to the academic stress experienced in general educational environments. The nursing training involves mastering theoretical and technical knowledge and evidence based research, and undertaking professional placements. These placements allow nurse students to translate their theoretical knowledge into practical skills. Although the practicums may commence with student nurses observing senior nurses, they move to advance levels, which involve the trainees interacting with multidisciplinary teams and patients in order to offer services and to engage in complex decision making. In order to graduate, the nurses have to successfully pass the theoretical and practical components of their training. Therefore, for international nursing students, it is important that they are able to express themselves authentically and accurately, as they must negotiate both an academic environment as well as their placement environment. However, the relationship between SLA and international nursing student stress remains almost unknown in the current 
literature. To better understand international nursing students' stress, the present paper sought to explore how demographic, cognitive, acculturative, and SLA factors are associated with the stress these students experience in academic and placement environments.

\section{LITERATURE REVIEW}

\section{International Nursing Students' Stress}

For nursing students, stress can be categorised as academic and placement-related stress. Nursing students have reported that issues related grasping complex concepts,, presentations, style of instruction, work load and passing written and oral assessments are some of the academic -related sources of stress that make their coursework challenging (Gibbons et al., 2009a; Higginson, 2006). In contrast, placement-related stress involves various sources of pressure from their course requirements to practice in actual hospital settings. Issues related to conducting clinical procedures, relationships with clinical staff and patients, and expectations of placements are some of the placement-related stressors that have been identified by nursing students (Gibbons et al., 2009a; Higginson, 2006).

In Australia, the majority of international nursing students are from Asia and they have English as their second language. Emerging literature is highlighting the challenges associated with using a second language for academic pursuits (Phakiti et al., 2013). Second language issues have been associated with academic-based difficulties in international students (Trice \& Yoo, 2007). International students require greater effort to overcome difficulties in their learning environment compared to domestic students (Andrade, 2006). In the case of nursing courses, second language can have a significant negative impact on international nursing students' learning of the subject content and successfully passing written exams (Phakiti et al., 2013). Additionally, international students in nursing programs can encounter professional and relational difficulties due to their communication in a second language. For students in the placement settings, relationships with patients and colleagues, as well as feelings of being a valuable member of the profession, can be negatively affected by SLA (Crawford \& Candlin, 2013). Subsequently, international students have been found to exhibit symptoms of worry, anxiety, stress, and depression with reference to their academic accomplishments (Bayram \& Bilgel, 2008; Brown, 2008). In spite of the significance of second language in learning experiences of international nursing students, the characteristics that make these students susceptible to stress with the academic-related and placement-related components of their course are not fully understood. 


\section{Demographic characteristics and stress}

While some demographic factors such as social support have been clearly linked with students' stress (Poyrazli et al., 2004), the effect of other demographic characteristics such as gender, age, and employment status have been less clear. Furthermore, there is scarce information on the demographic characteristics that make international nursing students vulnerable to program-specific stressors as well as SLA. With nursing programs consisting of a variety of mature-aged students who may possess prior professional nursing experience, and younger, inexperienced students, a few studies have examined the effect of age on other aspects of nursing student functioning such as academic achievement and styles of coping (Admi, 1997; Blackman, 2001). However, even this evidence has been conflicting (Blackman et al., 2007). It has been found that differences in financial satisfaction, employment status, and gender, are related to levels of reported distress in the general international student population (Khawaja \& Duncanson, 2008). In particular, females and those in full-time employment were found to report higher levels of depression. Yet other studies have shown that males were more likely to report feelings of loneliness (Poyrazli et al., 2004). With the absence of information on demographic effects on SLA, particularly with international nursing students, it would therefore be important for researchers to further extract information regarding the demographic characteristics that predispose international students to stress and SLA.

\section{Cognitive variables and stress}

Many studies have been conducted on the relationship between various cognitive variables and stress in the university student population. Aspects of personality, cognitive style, and dominant affect can also influence the amount of anxiety experienced by the second language speaker, with consequences on other areas of functioning (Bailey et al., 2000; Zheng, 2008).

\section{Fear of Negative Evaluation}

Research on language acquisition has suggested that fear of negative evaluation may mediate experiences of SLA (Casado \& Dereshiwsky, 2001; Cebreros, 2003). Cebreros' (2003) found that a fear of negative evaluation by peers and instructors was amongst the reported experiences of speaking anxiety, which also included avoidance of communicating in English as second language, and feelings of being overwhelmed, nervous, and confused. However, the evidence for fear of negative evaluation in SLA remains scarce. Additionally, as issues with fear of negative evaluation have also been found in nursing students, it would be crucial to explore further 
how this factor exacerbates SLA in a way that could influence performances of nursing students, particularly in placement settings where communication is inevitable.

\section{Perfectionism}

Perfectionism refers to critical self-evaluations based on high personal standards of performance and achievement (Frost et al., 1990), which can be adaptive or maladaptive. As has been found with international students, perfectionistic individuals tend to ruminate and worry about meeting personal expectations, which are often unrealistic, resulting in stress and an over-exhaustion of their ability to cope (Khawaja \& Dempsey, 2007). In particular, maladaptive perfectionists demonstrate higher levels of concern over mistakes, and self-doubt, which leads to higher levels of depression (Lee \& Park, 2011). Similarly, Sturman, Flett, Hewitt and Rudolph (2009) found that students with a sense of self-worth that was contingent on perfectionism were more vulnerable to depression. They proposed that students who had a greater need for successful performance and approval to maintain their sense of self-worth were more likely to develop workaholic, goal-obsessed styles that could lead to burnout, exhaustion, and psychological distress. They further clarified that these students are specifically vulnerable in situations where failure or a low sense of achievement is experienced (Sturman et al., 2009), a view that is further supported by Neff and Vonk (2009).

While perfectionism has not been directly assessed in SLA studies, especially within the nursing population, it is likely that maladaptive perfectionistic tendencies could exacerbate the experience of SLA. International nursing students with high personal standards that are difficult to achieve are at an increased risk of developing psychological distress, particularly with the added challenge of having to function as a studentnurse practitioner in professional settings where second language is the main medium of communication.

\section{Acculturation and Stress}

Two major aspects of acculturative stress experienced by international students include marginalization and separation (Khawaja \& Dempsey, 2008). Separation is evident in individuals who don't value belonging to the host culture and instead hold on to their original culture, while marginalization is defined as a lack of social connectedness with the host culture as well as their original culture (Berry, 1997). Sullivan and Kashubeck-West (2015) found that international students had higher rates of marginalization and acculturative stress compared to immigrant groups, and established that these higher rates are due to unique factors relating to being 
an international student rather than demographic factors.

Diminished interaction and interest between international students and members of the host culture can maintain or even exacerbate maladjustment and stress in international students, particularly with communication issues (Andrade, 2006; Major, 2005). Developing meaningful connection between the international student and members of the host culture not only promotes cross-cultural understanding (Ujitani \& Volet, 2008), but may also encourage second language communication in international students, which if avoided can lead to loss of self-confidence and self-efficacy, anxiety, sadness, fatigue, and feelings of inadequacy over their language skills (Brown, 2008; Major, 2005). As support between international students and members of the host culture, especially within the university, has been found to encourage the development of effective coping skills in nursing students (Seyedfatemi et al., 2007), understanding how acculturative stress relates to SLA can aid in developing management strategies for SLA and other aspects of international student stress.

\section{Second Language Anxiety (SLA)}

SLA refers to a performance-related anxiety, which can impact the students' well-being and academic achievement (Crawford \& Candlin, 2013). The physiological symptoms of SLA such as increased heart rate, hot flushes, perspiration, mind going blank, and a decreased ability to concentrate, are consistent with the symptoms of anxiety disorders (American Psychiatric Association, 2000; Andrade \& Williams, 2009). The cognitive symptoms, which include negative thoughts and worries about test performance, and stress about skill-based activities, can hinder learning (Andrade \& Williams, 2009). Social and emotional symptoms include feelings of shame and embarrassment, sadness, and frustration associated with expressing oneself and not being understood by native speakers (Brown, 2008).

Correlations have been found between written language proficiency and adjustment outcomes for international students (Andrade, 2006), however others have proposed that it is the spoken aspect that is most stressful part of the second language experience (Brown, 2008). It is important to note that much of SLA appears to be perpetuated by perceived competence rather than actual proficiency in the language (Brown, 2008; Casado \& Dereshiwsky, 2001; Khawaja \& Dempsey, 2007; MacIntyre et al., 1997; Pitts, 2009). Despite having met university language proficiency requirements, Brown's (2008) interview with international postgraduate students indicated perceived oral ability was still a major concern and contributor to the students' level of distress and embarrassment. This was reinforced by diminished contact between international and domestic 
students. International students tended to withdraw from situations of communication in the host language, while domestic students avoided or were less inclined to befriend international students as they perceived the effort required to modify their communication style as exhaustive (Brown, 2008). As such, studies like Brown's (2008) have found that second language issues in international universities can generate feelings of anxiety, frustration, sadness, fatigue, shame, withdrawal from social contact with the host culture, and avoidance of communicating in the host language. This leads to a cycle of diminished opportunities for reparative experiences that could otherwise habituate international students to anxiety-provoking and stressful interactions within academic and social settings.

\section{The Current Study}

Even though there have been some studies examining the relationship between demographic and cognitive factors of stress in international students (Khawaja \& Dempsey, 2007), there have been few studies examining the relationship between these stress factors and second language anxiety. Furthermore, there is a scarcity of research investigating the relationship between second language anxiety and stress in international nursing students as manifested in academic and placement settings. Hence, the current study aimed to explore the predictability that spoken and written SLA would add to international nursing student stress after taking into account the demographic, cognitive, and acculturative factors. It is hoped that the exploratory findings from this study would contribute to current literature on SLA and international nursing student stress, providing more in-depth understanding of the factors of international nursing student stress. This may influence future directions in industrial and institutional policies, in particular those pertaining to international nursing student wellbeing, as well as in the field of language psychology.

\section{Participants}

\section{RESEARCH METHOD}

One hundred and fifty two international students (females: 129, males: 23), enrolled in undergraduate nursing programs in faculties of nursing or midwifery across Australia participated in the study. The mean age of participants was 26.23 years $(S D=5.60)$. The majority of students were in their third year of study $(38.7 \%)$, while $32 \%$ were in their second year and $29.3 \%$ were in their first year. Majority of the participants spent between 1 - 3 years in Australia (42.1\%), while 23\% had been in Australia for one year or less, and $34.9 \%$ had been in Australia for three or more years. A mean of 10.31 years of studying English as a second language ( $S D$ $=6.69$ ) was also reported by the participants. 


\section{Measures}

The electronic survey consisted of a brief demographic questionnaire and six measures which have been widely used in international student research:

\section{Demographic measure}

Demographic and general information including age, gender, marital status, country of birth, current university level, and duration of study of the English language, were obtained through a form.

Learning \& Teaching-related and Placement-related Sources of Stress (Abridged form) (Gibbons et al., 2009a; Gibbons et al., 2009b)

An abridged version consisting of 6 placement-related stress items and 11 academic g-related items was used in the present study. Using a 6 point Likert scale $(0=$ Not at all to $6=$ Extremes source of stress $)$ the nursing students indicate their sources of stress (Gibbons et al., 2009b). Placementrelated items measure situations that can be a source of stress (e.g. learning on placements; expectations to practice nursing exceptional skills; being able to demonstrate adequate skills, when questioned by the supervisor and; building and sustaining a relationship with patients). Learning and teachingrelated items covered academic stress (e.g. being able to present at tutorials, able to participate in class discussion, expected to grasp concepts and abilty to analyse and evaluate subject matetr0. Statistical procedures were used by the authors to draw items out of their original 29-items scale with three subscales: learning \& teaching-related, placement-related, and course organisation-related sources of stress (Gibbons et al., 2009a). The original scale was found to have good test-retest reliability $(r=.8)$ (Gibbons et al., 2009a). Higher scores indicated a higher level of stress.

Second Language Speaking Anxiety Scale (SLSAS) (Woodrow, 2006).

This 12-item scale was developed to measure in-class and out-ofclass experience by English-as-a-second-language students. On a five point Likert scale $(1=$ Not at all anxious to $5=$ Most anxious $)$ the respondents indicate the level of anxiety associated with speaking in English language in an academic setting such as when participating in a formal class discussion, presenting in a class discussion, or asking the lecturer a question (e.g. The tutor/lecturer asks me a question in English in class). Items were modified to make them suitable for the present study. "Teacher" was changed to "Lecturer/ supervisor" and "school" was changed to "university/clinical placement setting". The author reported high reliability of the combined scales $(r=.94)$, the in-class subscale $(r=.89)$, and out-of-class subscale $(r$ $=.87$ ). High scores indicated a high level of anxiety associated with speaking English in academic setting. 
Second Language Writing Anxiety Inventory (SLWAI) (Cheng, 2004).

This 22-item scale measures second language writing anxiety. Respondents on a five point Likert scale ( $1=$ Strongly disagree to $5=$ Strongly agree) indicate the level of their somatic anxiety (e.g. I feel my heart pounding when I write English compositions under time constraint), cognitive anxiety (e.g. While writing English compositions, I feel worried and uneasy if I know they will be evaluated), and avoidance behaviour (e.g. I usually do my best to avoid writing English compositions), in relation to writing in English language. The scale is internally consistent (Cronbach's $\alpha$ $=.91)$. High scores indicated a high level of anxiety associated with writing in English for academic tasks.

Dysfunctional Perfectionism subscale (DYS-P) of The Frost Multidimensional Perfectionism Scale - Revised (FMPRS-R) (Khawaja \& Armstrong, 2005).

This 11-item subscale was used to measure pathological or maladaptive perfectionism traits characterised by rigid goal setting, excessive high standards, inability to experience a sense of fulfilment, and distress over personal capabilities (e.g. If I fail at university or work, I am a failure as a person). Items were rated on a five-point Likert scale from 1 (strongly disagree) to 5 (strongly agree). The author reported the scale to be internally consistent (Cronbach's $\alpha=.91$ ). Higher score indicated an elevated level of maladaptive perfectionism.

Brief Fear of Negative Evaluation Scale (Leary, 1983). This 12-item scale has been used to measure apprehension about being evaluated negatively by others. Respondents on a five-point Likert scale $(1=$ Not at all characteristic of me to $5=$ Extremely characteristic of me) indicate their fears of being judged by others (e.g. I worry about what other people will think of me even when I know it doesn't make any difference; I am frequently afraid of other people noticing my shortcomings). This scale was reported by the author to have good reliability (Cronbach's $\alpha=.90$ ). Higher score indicated a high level of negative evaluative fears.

Marginalisation \& Separation (Barry, 2001).

The marginalisation and separation subscales of the East Asian Acculturation Measure (EAAM) were used in the current study to assess maladaptive acculturation. The marginalisation and separation subscales consist of 16 items rated on a seven-point Likert scale ( 1 = strongly disagree to $7=$ strongly agree). Items reflected a tendency to interact with one's own culture by separating from the dominant culture (e.g. I prefer going to social gatherings where most of the people are from my culture) or a tendency to marginalise from dominant and other minority groups (e.g. I sometimes feel that neither Australians nor people from my culture like me). For the current study, items were reworded for relevance to the population such that the 
word "Asian" was rephrased as "a person from my culture", and "American" was replaced with "Australian". Barry (2001) reported the reliability of the marginalisation subscale to be good (Cronbach's $\alpha=.85$ ) and found the reliability rating of the separation subscale to be satisfactory (Cronbach's $\alpha=.76$ ). Higher scores indicated an elevated level of marginalisation and separation.

\section{Procedure}

Ethical clearance was obtained from the respective university. Administrators of Nursing Schools at 20 Australian universities were emailed information about the study and invited to participate in the study. They were requested to email the electronic flyer and information sheets about the present study to their undergraduate international nursing students. Those who were international nursing students, and used English as a second language, were invited to participate in the study via an electronic link to the study's online questionnaire, which took approximately 30 minutes to complete. Participation in the study was voluntary and nonidentifiable. Submission of the completed electronic survey was considered as informed consent. Response rate could not be calculated as there was no way to knowing, how many schools participated in the study. Further, there was no information about the number of students who received an invitation to the study.

\section{RESULTS}

\section{Preliminary analyses}

Statistical analyses were conducted using Statistical Package for the Social Sciences (SPSS) 22.0. The data set was examined and participants (n $=8$ ) who did not meet the criteria of being enrolled as an international nursing students and using English as a second language, were excluded from the analyses. Missing data were random and minimum. Items with less than 5\% missing data were replaced via mean imputation, yielding a final sample size of 152. Data screening procedures also included checks for normality and other statistical assumptions. All statistical assumptions were met and multicollinearity was not an issue. The internal consistency of all the scales was measured by using Cronbach alpha. The internal consistency for Learning and Teaching-related and Placement-related Sources of Stress (.90), Second language Speaking Anxiety Scale (.94), Second Language Writing Anxiety Inventory (.90), Dysfunctional Perfectionism scale subscale (.88), Brief Fear of Negative Evaluation (.84), and Marginalisation (.89) and Separation (.77) were satisfactory.

Bivariate correlations were used to examine the relationship among the variables. As seen by the table, an increase in the years spent in studying English were related to reduction in marginalisation and feelings of anxiety 
linked with speaking and writing in a second language. There was moderate relationship among a sense of being separated, marginalised, dysfunctional perfectionism, anxiety related with the spoken and written second language, academic and placement stress. Finally, academic stress was highly associated with placement stress.

\begin{tabular}{|c|c|c|c|c|c|c|c|c|c|c|c|c|}
\hline Variables & 1 & 2 & 3 & 4 & 5 & 6 & 7 & 8 & 9 & 10 & 11 & 12 \\
\hline 1. Age & & -.09 & -.00 & -.05 & -.01 & -.04 & -.07 & -.10 & .02 & -.10 & .10 & .01 \\
\hline 2. YrsAus & & & $.39 * *$ & .09 & -.14 & .01 & -.00 & -.07 & -.13 & .00 & -.05 & -.14 \\
\hline 3. Uni level & & & & -.02 & .10 & -.06 & .10 & -.15 & -.11 & -.03 & -.05 & -.07 \\
\hline 4. Years Eng Study & & & & & -.11 & $-.18^{*}$ & .00 & -.05 & $-.20^{*}$ & $-.21^{*}$ & -.13 & -.07 \\
\hline 5. EAAMSep & & & & & & $.44 * *$ & .12 & $.19^{*}$ & $.38^{* *}$ & $.44^{* *}$ & $.23^{* *}$ & .13 \\
\hline 6. EAAMMAR & & & & & & & $.24 * *$ & $.39 * *$ & $.41^{* *}$ & $.42 * *$ & $.41^{* *}$ & $.24^{* * *}$ \\
\hline 7. DYS-P & & & & & & & & $.55^{* *}$ & $.16^{*}$ & $.19 *$ & $.25^{* *}$ & $.17^{*}$ \\
\hline 8. FNE & & & & & & & & & $.31^{* * *}$ & $.22 * *$ & $.34 * *$ & $.25^{* *}$ \\
\hline 9. SLS & & & & & & & & & & $.53^{* *}$ & $.60^{* *}$ & $.45^{* * *}$ \\
\hline 10. SLW & & & & & & & & & & & $.44 * *$ & $.30^{* *}$ \\
\hline 11. Academic Stress & & & & & & & & & & & & $.79 * *$ \\
\hline
\end{tabular}

Note: YrsAus: Years in Australia; Uni Level: year of enrolment; EAAMMAR: EAAM Marginalisation subscale; EAAMSep: EAAM Separation subscale; DYS-P: Dysfunctional Perfectionism subscale; FNE: Fear of Negative Evaluation; SLS: Second Language Anxiety -Spoken subscale; SLW: Second Language Anxiety - Writing subscale; *: correlations significant at .05 level; **: correlations significant at .01 level.

\section{Relationship between demographic, cognitive, acculturative, and language-related variables with stress in international nursing students} Hierarchical multiple regression was used to identify how well the demographic, cognitive, acculturative, and language-related measures predicted levels of academic-related stress, and placement-related stress, in international nursing students. The dependent variables were academic stress (measures by learning and teaching-related sources of stress subscale) for the first hierarchical regression analysis, and placement-related sources of stress (measured by placement-related sources of stress subscale) for the second hierarchical regression analysis. The predictor variables for both analyses were the seven demographic factors (age, sex, years spent in Australia, years spent studying English as a second language, employment status, relationship status, and university level), two cognitive measures (fear of negative evaluation and dysfunctional perfectionism), two acculturation measures (marginalization and separation), and two languagerelated measures (spoken SLA and written SLA). Continuous demographic variables were re-computed as categorical variables, such that two categories were obtained for age, while three categories were obtained for years spent in Australia and years spent studying English as second language.

With academic-related sources of stress as the dependent variable, the demographic variables were entered in Step 1 of the hierarchical regression, which did not explain a significant proportion of variance, $R_{\text {change }}^{2}=.05, F_{\text {change }}(7,136)=.94, p>.05$. At Step 2, dysfunctional perfectionism and fear of negative evaluation were added. A significant change was found, $R_{\text {change }}^{2}=.12, F_{\text {change }}(2,134)=9.40, p<.01$. Only fear of 
Table 2: Hierarchical multiple regression of demographic, cognitive, acculturative, and SLA factors on academic-related stress

\begin{tabular}{|c|c|c|c|c|c|c|}
\hline & & $b$ & SE $B$ & $\beta$ & $p$ & $95 \% \mathrm{CI}$ \\
\hline \multirow[t]{8}{*}{$\overline{\text { Step } 1}$} & (Constant) & 29.122 & 7.140 & & .000 & $15.00,43.24$ \\
\hline & AgeCAT & 1.699 & 1.866 & .083 & .364 & $-1.99,5.39$ \\
\hline & YrsAus & .977 & 1.355 & .072 & .472 & $-1.70,3.66$ \\
\hline & Sex & 4.586 & 2.486 & .158 & .067 & $-.330,9.50$ \\
\hline & StatusRC & -1.209 & 1.150 & -.100 & .295 & $-3.48,1.07$ \\
\hline & UniLevel & -.830 & 1.158 & -.067 & .475 & $-3.12,1.46$ \\
\hline & Employment & .622 & 1.086 & .051 & .568 & $-1.53,2.77$ \\
\hline & YearsEngStudy & -1.631 & 1.066 & -.132 & .128 & $-3.74, .48$ \\
\hline \multirow[t]{10}{*}{ Step 2} & (Constant) & 11.197 & 7.904 & & .159 & $-4.44,26.83$ \\
\hline & AgeCAT & 2.830 & 1.782 & .138 & .115 & $-.70,6.36$ \\
\hline & YrsAus & .792 & 1.280 & .058 & .537 & $-1.74,3.32$ \\
\hline & Sex & 4.087 & 2.348 & .141 & .084 & $-.56,8.73$ \\
\hline & StatusRC & -.966 & 1.087 & -.080 & .376 & $-3.12,1.18$ \\
\hline & UniLevel & -.460 & 1.105 & -.037 & .678 & $-2.65,1.73$ \\
\hline & Employment & .673 & 1.037 & .055 & .518 & $-1.38,2.72$ \\
\hline & YearsEngStudy & -1.403 & 1.007 & -.114 & .166 & $-3.40, .59$ \\
\hline & DYS-P & .222 & .124 & .174 & .075 & $-.02, .47$ \\
\hline & FNE & .277 & .124 & .222 & .027 & $.03, .52$ \\
\hline \multirow[t]{12}{*}{ Step 3} & (Constant) & 10.667 & 7.745 & & .171 & $-4.65,25.99$ \\
\hline & AgeCAT & 1.221 & 1.750 & .060 & .487 & $-2.24,4.68$ \\
\hline & YrsAus & .389 & 1.233 & .029 & .753 & $-2.05,2.83$ \\
\hline & Sex & 3.404 & 2.245 & .117 & .132 & $-1.04,7.85$ \\
\hline & StatusRC & -.597 & 1.041 & -.050 & .567 & $-2.66,1.46$ \\
\hline & UniLevel & -.157 & 1.057 & -.013 & .882 & $-2.25,1.93$ \\
\hline & Employment & .926 & .991 & .076 & .351 & $-1.03,2.89$ \\
\hline & YearsEngStudy & -.553 & .985 & -.045 & .575 & $-2.50,1.39$ \\
\hline & DYS-P & .165 & .119 & .130 & .166 & $-.07, .40$ \\
\hline & FNE & .123 & .124 & .098 & .326 & $-.12, .37$ \\
\hline & EAAMSep & .008 & .105 & .006 & .941 & $-.20, .21$ \\
\hline & EAAMMar & .317 & .089 & .341 & .001 & $.14, .49$ \\
\hline \multirow[t]{14}{*}{$\overline{\text { Step } 4}$} & (Constant) & 2.050 & 6.932 & & .768 & $-11.67,15.76$ \\
\hline & AgeCAT & .579 & 1.481 & .028 & .697 & $-2.35,3.51$ \\
\hline & YrsAus & .684 & 1.057 & .050 & .518 & $-1.41,2.78$ \\
\hline & Sex & 2.827 & 1.897 & .097 & .139 & $-.93,6.58$ \\
\hline & StatusRC & -.960 & .882 & -.080 & .278 & $-2.71, .79$ \\
\hline & UniLevel & .025 & .893 & .002 & .978 & $-1.74,1.79$ \\
\hline & Employment & .850 & .838 & .070 & .312 & $-.81,2.51$ \\
\hline & YearsEngStudy & .406 & .847 & .033 & .632 & $-1.27,2.08$ \\
\hline & DYS-P & .195 & .100 & .153 & .055 & $-.004, .39$ \\
\hline & FNE & .002 & .106 & .001 & .987 & $-.21, .21$ \\
\hline & EAAMSep & -.174 & .094 & -.143 & .066 & $-.36, .01$ \\
\hline & EAAMMar & .179 & .078 & .193 & .023 & $.03, .33$ \\
\hline & SLS & .465 & .074 & .511 & .000 & $.32, .61$ \\
\hline & SLW & .076 & .062 & .103 & .221 & $-.05, .20$ \\
\hline
\end{tabular}

Note: YrsAus: Years in Australia; StatusRC: relationship status; Unilevel: enrolment year at the university; DYS-P: Dysfunctional Perfectionism subscale ; FNE: Fear of Negative Evaluation; EAAMMAR: EAAM Marginalisation subscale; EAAMSep: EAAM Separation subscale; SLS: Second Language Anxiety -Spoken subscale; SLW: Second Language Anxiety - Writing subscale. 
Journal of International Students, 7(3) 2017

Table 3: Hierarchical multiple regression of demographic, cognitive, acculturative, and SLA factors on placement-related stress

\begin{tabular}{|c|c|c|c|c|c|c|}
\hline & & $b$ & SE $B$ & $\beta$ & $p$ & $95 \% \mathrm{CI}$ \\
\hline \multirow[t]{8}{*}{ Step 1} & (Constant) & 11.276 & 4.096 & & .007 & $3.18,19.38$ \\
\hline & AgeCAT & 1.259 & 1.071 & .107 & .242 & $-.86,3.38$ \\
\hline & YrsAus & -.121 & .777 & -.015 & .876 & $-1.66,1.42$ \\
\hline & Sex & 2.645 & 1.426 & .158 & .066 & $-.17,5.47$ \\
\hline & StatusRC & -.775 & .660 & -.112 & .242 & $-2.08, .53$ \\
\hline & UniLevel & -.340 & .664 & -.047 & .610 & $-1.65, .97$ \\
\hline & Employment & .693 & .623 & .098 & .268 & $-.54,1.93$ \\
\hline & YearsEngStudy & -.459 & .611 & -.064 & .455 & $-1.67, .75$ \\
\hline \multirow[t]{10}{*}{ Step 2} & (Constant) & 3.900 & 4.686 & & .407 & $-5.37,13.17$ \\
\hline & AgeCAT & 1.725 & 1.057 & .146 & .105 & $-.37,3.82$ \\
\hline & YrsAus & -.197 & .759 & -.025 & .795 & $-1.70,1.30$ \\
\hline & Sex & 2.440 & 1.392 & .146 & .082 & $-.31,5.19$ \\
\hline & StatusRC & -.675 & .644 & -.097 & .297 & $-1.95, .60$ \\
\hline & UniLevel & -.187 & .655 & -.026 & .776 & $-1.48,1.11$ \\
\hline & Employment & .713 & .615 & .101 & .248 & $-.50,1.93$ \\
\hline & YearsEngStudy & -.365 & .597 & -.051 & .542 & $-1.55, .82$ \\
\hline & DYS-P & .091 & .073 & .124 & .217 & $-.05, .24$ \\
\hline & FNE & .114 & .073 & .159 & .121 & $-.03, .26$ \\
\hline \multirow[t]{12}{*}{ Step 3} & (Constant) & 4.795 & 4.683 & & .308 & $-4.47,14.06$ \\
\hline & AgeCAT & .950 & 1.058 & .080 & .371 & $-1.14,3.04$ \\
\hline & YrsAus & -.469 & .745 & -.060 & .531 & $-1.94,1.01$ \\
\hline & Sex & 2.126 & 1.358 & .127 & .120 & $-.56,4.81$ \\
\hline & StatusRC & -.501 & .629 & -.072 & .428 & $-1.75, .74$ \\
\hline & UniLevel & -.069 & .639 & -.010 & .915 & $-1.33,1.20$ \\
\hline & Employment & .839 & .599 & .119 & .164 & $-.35,2.02$ \\
\hline & YearsEngStudy & .008 & .595 & .001 & .989 & $-1.17,1.19$ \\
\hline & DYS-P & .067 & .072 & .091 & .353 & $-.08, .21$ \\
\hline & FNE & .043 & .075 & .059 & .572 & $-.11, .19$ \\
\hline & EAAMSep & -.063 & .063 & -.090 & .321 & $-.19, .06$ \\
\hline & EAAMMar & .167 & .054 & .313 & .002 & $.06, .27$ \\
\hline \multirow[t]{14}{*}{ Step 4} & (Constant) & .699 & 4.501 & & .877 & $-8.21,9.60$ \\
\hline & AgeCAT & .638 & .961 & .054 & .508 & $-1.26,2.54$ \\
\hline & YrsAus & -.320 & .686 & -.041 & .642 & $-1.68,1.04$ \\
\hline & Sex & 1.847 & 1.232 & .110 & .136 & $-.59,4.29$ \\
\hline & StatusRC & -.678 & .573 & -.098 & .238 & $-1.81, .46$ \\
\hline & UniLevel & .019 & .580 & .003 & .974 & $-1.13,1.17$ \\
\hline & Employment & .803 & .544 & .114 & .142 & $-.27,1.88$ \\
\hline & YearsEngStudy & .468 & .550 & .066 & .396 & $-.62,1.56$ \\
\hline & DYS-P & .081 & .065 & .111 & .215 & $-.05, .21$ \\
\hline & FNE & -.016 & .069 & -.022 & .816 & $-.15, .12$ \\
\hline & EAAMSep & -.150 & .061 & -.214 & .015 & $-.27,-.03$ \\
\hline & EAAMMar & .101 & .051 & .189 & .048 & $.001, .20$ \\
\hline & SLS & .226 & .048 & .431 & .000 & $.13, .32$ \\
\hline & SLW & .035 & .040 & .081 & .389 & $-.05, .11$ \\
\hline
\end{tabular}

Note: YrsAus: Years in Australia; StatusRC: relationship status; Unilevel: enrolment year at the university; DYS-P: Dysfunctional Perfectionism subscale ; FNE: Fear of Negative Evaluation; EAAMMAR: EAAM Marginalisation subscale; EAAMSep: EAAM Separation subscale; SLS: Second Language Anxiety -Spoken subscale; SLW: Second Language Anxiety - Writing subscale. 
negative evaluation was statistically significant, as seen in Table 2. At Step 3 separation and marginalisation were added to the model, and a significant change was found, $R_{\text {change }}^{2}=.09, F_{\text {change }}(2,132)=7.70, p<.001$. As seen in Table 2, fear of negative evaluation was no longer significant, with marginalisation being the only significant contributor to the model at this step. At Step 4, the language related predictors of spoken SLA and written SLA were entered into the model, and a significant change was found, $R_{\text {change }}^{2}=.22, F_{\text {change }}(2,130)=27.59, p<.001$. The total model explained $47.4 \%$ of the variance in placement-related stress in international nursing students (adjusted $\left.\mathrm{R}^{2}=.42\right), F(13,130)=9.012, \mathrm{p}<.001$. Marginalisation remained a significant contributor to the model, with spoken SLA the only other significant contributor to the model.

Another hierarchical regression was performed with placementrelated sources of stress as the dependent variable, and the same predictor variables entered in the same steps as the previous hierarchical regression analysis. At Step 1, the demographic variable were entered with no significant prediction in the variance, $R_{\text {change }}^{2}=.06, F_{\text {change }}(7,136)=1.14, p$ $>.05$. At Step 2, no significant change was found when the cognitive variables were entered, $R_{\text {change }}^{2}=.06, F_{\text {change }}(2,134)=4.53, p>.05$. At Step 3 , the acculturation measures of separation and marginalisation were entered, and a significant change was found, $R_{\text {change }}^{2}=.06, F_{\text {change }}(2,132)=$ $4.86, p<.01$. Only marginalisation was a significant measure, as seen in Table 2. At Step 4, spoken SLA and written SLA were entered into the model, and a significant change was found, $R_{\text {change }}^{2}=.16, F_{\text {change }}(2,130)=$ $15.28, p<.001$. The total model explained $33.3 \%$ of the variance in placement-related stress in international nursing students (adjusted $\mathrm{R}^{2}=$ $.27), F(13,130)=4.99, \mathrm{p}<.001$.

\section{DISCUSSION AND CONCLUSIONS}

The current study examined the demographic, cognitive, acculturative, and language characteristics of international nursing students that are associated with their stress in both academic and placement environments. The hypothesis that after taking into account demographic, cognitive, and acculturative factors, spoken and written SLA would add to the predictability of international nursing student stress was partially supported. For both sources of nursing student stress, spoken SLA was a significant measure in the total prediction model, while written SLA was not. This is consistent with Brown's (2008) study where international students most often cited distress with spoken second language communication as a problem. It appears that international students' anxiety related experiences associated with spoken second language may inhibit them from interacting and communicating with the members of the host society (Andrade, 2006; 
Major, 2005). Consequently, this type of avoidance can prevent them from addressing their negative expectations and fears. It also precludes them from enhancing their skills and coping strategies (Seyedfatemi et al., 2007).

Marginalization was also a significant measure in predicting stress in both the academic and the placement environments. The finding that a marginalization acculturative style is positively related to experiencing stress supports the findings of Sullivan and Kashubeck-West (2015), who found that factors relating to being an international student were the cause for higher rates of marginalization in this population. Factors such as being in a new cultural environment where there is pressure to perform well academically, as well as dealing with isolation and distance from family and friends, could result in higher levels of stress in both of these environments (Sullivan \& Kashubeck-West, 2015).

It is interesting to note that there was a significant negative relationship between separation and placement-related sources of stress, but not for academic-related stress. From this relationship it can be inferred that those who experience high levels of stress in the placement environment are unlikely to have an acculturative style similar to separation. While causality cannot be assumed, it can be hypothesized that the communication in a placement environment, which is essential in order to successfully complete the tasks required, can assist students to form relationships with members of the host culture. These relationships may then act as a protective factor, making it less likely for students to reject the host culture. Whereas, in academic environments there is less direct interaction with the host society, possibly explaining the lack of relationship between separation and this environment. This tentative finding mirrors other studies where it has been found that social support can help with the acculturation process (Major, 2005).

While previous studies with international student populations have found that fear of negative evaluation is a significant predictor of stress (Neff \& Vonk, 2009; Begley \& White, 2003; Roadebaugh et al., 2004), the results of this study revealed that fear of negative evaluation was only significant before acculturative factors were added to the model, and only in the academic environment. Academic stress may be highly influenced by socially based experiences like fear of negative evaluation and marginalization, where stressors may manifest more in the form of performance apprehension and fear of failing. These experiences are both based on social evaluation, compared to the pressure of clinic placements, which may be more oriented around self-appraisal. Although marginalization was significant in both environments, the Beta values show that marginalization was higher in the academic environment. As the current study assessed only the effects of the maladaptive dimensions of cognitive 
factors, both of which were found to be non-significant when accounted for by acculturative and SLA factors, it suggests that the influence of cognitive styles on international student stress may be greater when considered in tandem with their adaptive dimensions.

The finding that there was no significant relationship between demographic characteristics thought to predispose international nursing students to stress and the stress experienced in two nursing student environments was not altogether unexpected. The current literature offers conflicting results regarding the impact that demographic characteristics can have on student stress levels (Blackman et al., 2007), and this study provides further support for the suggestion that demographic factors do not play a role in student stress.

\section{IMPLICATIONS}

Overall, the findings reiterate the significance of helping international students who are working with a second language. At the university level, counselling services should introduce resources and interventions aimed at building resilience in international students. At the school level, nursing schools should factor in coping and management of second language issues into their programs, especially with respects to second language communication. Further, nursing school should introduce activities that promote international nursing students' interaction with the domestic students, as this would facilitate their proficiency and confidence with the second language. Finally, academics at the school level would have to be mindful of the second language anxiety while teaching or assessing these students. The supervisors at the placements should take into account the anxiety that may be associated with a nurse students' performance at a placement. Special programs that target the anxiety of speaking a second language in academic and clinical settings would be helpful for international students undertaking nursing degrees. There are several limitations to the current study. One of the limitations of the study is that no causal relationships could be concluded from the current analyses. Secondly, response rates could not be tracked and the sample size was relatively small. This could have contributed to the lack of significant findings of important demographic differences within the population. Thirdly, with the sample consisting solely of international students from Australia, there may be conditions unique to this population that limits its generalizability to international nursing students in other countries. Fourthly, there are no normative data available to compare the level of stress and SLA reported by the current sample and that of other international student populations. Lastly, apart from the stress scale, validation of the utilization of the other measures with the international nursing student population is still severely 
limited, and thus needs to be interpreted with caution. Also, the abridged version of the index of sources of nursing stress used in the current study has not been validated by other sources. As such, the results from the current study need to be interpreted with caution. Future research should include corrections for these limitations, particularly in terms of making comparisons with other international student populations and utilizing measures that have been well validated with the nursing student population.

In spite of the shortcomings, the findings from the current study do reveal important information about international students' academic stress and SLA. To the authors' knowledge, the study has presented for the first time that SLA, particularly the spoken aspect, is a significant factor in international nursing student stress, over and above the impact of other psychological factors that are currently associated with international student stress. Yet more research on SLA needs to be done, especially with prevalence and the lived experience of SLA. Future research adopting a qualitative method will be useful in explicating this phenomenon from a real-world perspective, possibly shedding light on how SLA manifests at an individual level and how it translates into internal experiences of the Self and of the Self in relation to others.

\section{REFERENCES}

American Psychiatric Association. (2000). Diagnostic and statistical manual of mental disorders (4th ed., text rev.). Washington, DC: Author.

Admi, H. (1997). Nursing students' stress during the initial clinical experience. Journal of Nursing Education, 36(7), 323-327.

Andrade, M. S. (2006). International students in English-speaking universities: Adjustment factors. Journal of Research in International Education, 5(2), 131-154. doi: 10.1177/1475240906065589

Andrade, M., \& Williams, K. (2009). Foreign language learning anxiety in Japanese EFL university classess: Physical, emotional, expressive, and verbal reactions. Sophia Junior College Faculty Journal, 29, 1-24.

Bailey, P., Onwuegbuzie, A. J., \& Daley, C. E. (2000). Correlates of anxiety at three stages of the foreign language learning process. Journal of Language and Social Psychology, 19(4), 474-490. doi: 10.1177/0261927X00019004005

Barry, D. T. (2001). Development of a new scale for measuring acculturation: The East Asian Acculturation Measure (EAAM). Journal of Immigrant Health, 3(4), 193-197.

Bayram, N., \& Bilgel, N. (2008). The prevalence and socio-demographic correlations of depression, anxiety and stress among a group of university students. Social psychiatry and psychiatric epidemiology, 43(8), 667-672.

Begley, C. M., \& White, P. (2003). Irish nursing students' changing self-esteem and fear of negative evaluation during their preregistration programme. Journal of Advanced Nursing, 42(4), 390-401. 
Berry, J.W. (1997) Immigration, acculturation, and adaptation. Applied Psychology: An International Review 46(1): 5-34. dor: 10.1111/j.14640597.1997.tb01087.x.

Blackman, I. (2001). A predictive model identifying latent variables, which influence undergraduate student nurses' achievement in mental health nursing skills. International Education Journal, 2(4), 53-64.

Blackman, I., Hall, M., \& Darmawan, I. G. N. (2007). Undergraduate nurse variables that predict academic achievement and clinical competence in nursing. International Education Journal, 8(2), 222-236.

Brown, L. (2008). Language and anxiety: An ethnographic study of international postgraduate students. Evaluation and Research in Education, 21(2), 7595.

Casado, M. A., \& Dereshiwsky, M. I. (2001). Foreign language anxiety of university students. College Student Journal, 35(4), 539-562.

Cebreros, A. M. O. (2005). Measuring language anxiety perceived by Spanish university students of English. Retrieved from http://www.publicacions.ub.es/revistes/bells12/articulos.asp?codart=102

Cheng, Y.S. (2004). A measure of second language writing anxiety: Scale development and preliminary validation. Journal of Second Language Writing, 13, 313-335.

Crawford,T. \& Candlin,S. (2013) A literature review of the language needs of nursing students who have English as a second/other language and the effectiveness of English language support programmes. Nurse Education in Practice 13(3): 181-185. doi: 10.1016/j.nepr.2012.09.008.

Fritz, M. V., Chin, D., \& DeMarinis, V. (2008). Stressors, anxiety, acculturation and adjustment among international and North American students. International Journal of Intercultural Relations, 32, 244-259. doi:10.1016/j.ijintrel.2008.01.001

Frost, R. O., Marten, P., Lahart, C., \& Rosenblate, R. (1990). The dimensions of perfectionism. Cognitive Therapy \& Research, 14(5), 449-468. doi: 01475916/90/1000-0449506.00/0

Gibbons, C., Dempster, M., \& Moutray, M. (2009a). Surveying nursing students on their sources of stress: A validation study. Nurse Education Today, 29, 867-872.

Gibbons, C., Dempster, M., \& Moutray, M. (2009b). Index of sources of stress in nursing students: A confirmatory factor analysis. Journal of Advanced Nursing, 65(5), 1095-1102.

Higginson, R. (2006). Fears, worries and experiences of first-year pre-registration nursing students: A qualitative study. Nurse Researcher, 13(3), 32-49.

Khawaja, N. G., \& Armstrong, K. A. (2005). Factor structure and psychometric properties of the Frost Multidimensional Perfectionism Scale developing shorter versions using an Australian sample. Australian Journal of Psychology, 57(2), 129-138.

Khawaja, N. G., \& Dempsey, J. (2007). Psychological distress in international university students: An Australian study. Australian Journal of Guidance \& Counselling, 17(1), 13-27.

Khawaja, N. G., \& Dempsey, J. (2008). A comparison of International and domestic 
tertiary students in Australia. Australian Journal of Guidance \& Counselling, 18(1), 30-46.

Khawaja, N. G., \& Duncanson, K. (2008). Using the university student depression inventory to investigate the effects of demographic variables on students' depression. Australian Journal of Guidance \& Counselling, 18(2), 1-15.

Leary, M. R. (1983). A brief version of the Fear of Negative Evaluation scale. Personality and Social Psychology Bulletin, 9(3), 371-375. doi: $10.1177 / 0146167283093007$

Lee, D.-G., \& Park, H.-J. (2011). Cross-cultural validity of the Frost Multidimensional Perfectionism scale in Korea. The Counseling Psychologist, 39(2), 320-346. doi: 10.1177/0011000010365910

MacIntyre, P.D., Noels, K.A., \& Clément, R. (1997) Biases in self-ratings of second language proficiency: The role of language anxiety. Language Learning 47(2): 265-287.

Major EM (2005) Co-national support, cultural therapy, and the adjustment of Asian students to an English-speaking university culture. International Education Journal 6(1): 84-95.

Major, E. M. (2005). Co-national support, cultural therapy, and the adjustment of Asian students to an English-speaking university culture. International Education Journal, 6(1), 84-95.

Neff, K. D., \& Vonk, R. (2009). Self-compassion versus global self-esteem: Two different ways of relating to oneself. Journal of Personality, 77(1), 23-50.

Phakiti, A., Hirsh, D., \& Woodrow, L. (2013) It's not only English: Effects of other individual factors on English language learning and academic learning of ESL international students in Australia. Journal of Research in International Education 12(3): 239-258. doi: 10.1177/1475240913513520.

Pitts, M. J. (2009). Identity and the role of expectations, stress, and talk in shortterm student sojourner adjustment: An application of the integrative theory of communication and cross-cultural adaptation. International Journal of Intercultural Relations, 33, 450-462.

Poyrazli, S., Kavanaugh, P. R., Baker, A., \& Al-Timimi, N. (2004). Social support and demographic correlates of acculturative stress in international students. Journal of College Counseling, 7(1), 73-82.

Seyedfatemi, N., Tafreshi, M., \& Hagani, H. (2007). Experienced stressors and coping strategies among Iranian nursing students. BMC Nursing, 6(1), 1111. doi: 10.1186/1472-6955-6-11

Sturman, E. D., Flett, G. L., Hewitt, P. L., \& Rudolph, S. G. (2009). Dimensions of Perfectionism and Self-worth Contingencies in Depression. Journal of Rational-Emotive \& Cognitive-Behavior Therapy, 27(4), 213.

Sullivan, C. \& Kashubeck-West,S. (2015) The interplay of international students' acculturative stress, social support, and acculturation modes. Journal of International Students, 5(1): 1-11.

Trice, A. G., \& Jin Eun, Y. (2007). International graduate students' perceptions of their academic experience. Journal of Research in International Education, 6(1), 41-66. doi: 10.1177/1475240907074788

Ujitani, E., \& Volet, S. (2008). Socio-emotional challenges in international education. Journal of Research in International Education, 7(3), 279-303. 
doi: $10.1177 / 1475240908099975$

Woodrow, L. (2006). Anxiety and speaking English as a second language. RELC Journal, 37(3), 308-328. doi: 10.1177/0033688206071315

$\mathrm{Yu}, \mathrm{B} .$, \& Shen, H. (in press). Predicting roles of linguistic confidence, integrative motivation and second language proficiency on cross-cultural adaptation. International Journal of Intercultural Relations. doi: 10.1016/j.ijintrel.2010.12.002

Zheng, Y. (2008). Anxiety and second/foreign language learning revisited. Canadian Journal for New Scholars in Education/Revue canadienne des jeunes chercheures et chercheurs en education, 1(1), 1-12.

NIGAR G KHAWAJA, PhD, is an Associate Professor at the School of Psychology and Counselling, Queensland University of Technology. She is a clinical psychologist and conducts research in clinical and transcultural psychology. She is the coordinator of the Master and Professional Doctorate in Clinical Psychology programs. Email: n.khawaja@qut.edu.au

SABRINA CHAN, DPsy is a clinical psychologist with clinical and transcultural research interest.

GEORGIA STEIN is currently completing her Master in Clinical Psychology. She is interested in clinical and transcultural research.

Manuscript submitted: February, 21, 2016

Manuscript revised: April 4, 2016

Accepted for publication: October 20, 2016 